\title{
SIX1 coordinates with TGF $\beta$ signals to induce epithelial-mesenchymal transition in cervical cancer
}

\author{
SHU-HUA SUN ${ }^{1 *}$, DAN LIU $^{2 *}$, YUN-TE DENG ${ }^{3 *}$, XIAO-XUE ZHANG ${ }^{2}$, \\ DONG-YI WAN ${ }^{2}$, BI-XIN XI $^{2}$, WEI HUANG ${ }^{1}$, QIAN CHEN ${ }^{1}$, MENG-CHEN LI $^{2}$, \\ MING-WEI WANG ${ }^{3}$, FEI YANG $^{3}$, PING SHU $^{4}$, KE-ZHI WU ${ }^{4}$ and QING-LEI GAO ${ }^{2}$ \\ ${ }^{1}$ Department of Gynecologic Oncology, Hubei Cancer Hospital, Wuhan, Hubei 430023; ${ }^{2}$ Cancer Biology Research Center, \\ Tongji Hospital, Tongji Medical College, Huazhong University of Science and Technology, Wuhan, Hubei 430074; \\ Departments of ${ }^{3}$ Pathology and ${ }^{4}$ Medical Records Room, Hubei Cancer Hospital, Wuhan, Hubei 430023, P.R. China
}

Received January 24, 2015; Accepted March 4, 2016

DOI: $10.3892 / 01.2016 .4797$

\begin{abstract}
Epithelial-mesenchymal transition (EMT) plays a critical role in promoting tumor invasion and metastasis. However, the key cofactors that modulate the signal transduction to induce EMT have note been fully explored to date. The present study reports that sine oculis homeobox homolog 1 (SIX1) is able to promote EMT of cervical cancer by coordinating with transforming growth factor (TGF) $\beta$-SMAD signals. The expression of SIX1 was negatively correlated with the expression of the epithelial marker E-cadherin in two independent groups of cervical cancer specimens. SIX1 could promote the transition of mesenchymal phenotype in the presence of active TGF $\beta$ signals in vitro and in vivo. TGF $\beta$-SMAD signals were required for the SIX1-mediated promotion of EMT and metastatic capacity of cervical cancer cells. Together, SIX1 and TGF $\beta$ cooperated to induce more remarkable changes in the transition of phenotype than each of them alone, and coordinated to promote cell motility and tumor metastasis in cervical cancer. These results suggest that the coordination of SIX1 and TGF $\beta$ signals may be crucial in the EMT program, and that SIX1/TGF $\beta$ may be considered a valuable marker for evaluating the metastatic potential of cervical cancer cells, or a therapeutic target in the treatment of cervical cancer.
\end{abstract}

\section{Introduction}

The transdifferentiation of epithelial cells into motile mesenchymal cells, a developmental program termed

Correspondence to: Dr Qing-Lei Gao, Cancer Biology Research Center, Tongji Hospital, Tongji Medical College, Huazhong University of Science and Technology, 1095 Jiefang Avenue, Wuhan, Hubei 430074, P.R. China

E-mail: qingleigao@hotmail.com

*Contributed equally

Key words: SIX1, TGF $\beta$, EMT, metastasis, cervical cancer epithelial-mesenchymal transition (EMT), has been demonstrated to play a critical role in promoting tumor invasion and metastasis $(1,2)$. The functional hallmark of the EMT program is to endow stationary epithelial cells with migratory and invasive ability (2). Thus, the transition into a mesenchymal phenotype is considered as a marker of metastatic potential in numerous epithelium-derived carcinomas, including cervical cancer $(3,4)$.

The EMT program is triggered and controlled by multiple signaling pathways that are activated in response to extracellular stimuli $(5,6)$. Among these, the transforming growth factor (TGF) $\beta$ signaling pathway has a predominant role in promoting epithelial plasticity that may progress to EMT (6-8). However, a large number of non-invasive tumor cells did not undergo TGF $\beta$-induced EMT in vitro (9), suggesting that TGF $\beta$ may not be able to efficiently activate signaling pathways in non-invasive tumor cells. It has been previously observed that the effect of TGF $\beta 1$ may be enhanced by other factors that could cooperate with TGF $\beta 1$ to induce sufficient activation of multiple signaling pathways (10). While extracellular factors may cooperate with $\operatorname{TGF} \beta(7,8)$, it remains unclear whether the increased expression of transcription factors in tumor cells could increase the sensitivity of tumor cells to TGF $\beta$ stimulation and promote TGF $\beta$-induced EMT.

Sine oculis homeobox homolog 1 (SIX1) is a transcription factor associated with development that is rarely expressed in the majority of adult tissues $(11,12)$. However, overexpression of SIX1 has been observed in various malignancies, and frequently correlates with poor clinical prognosis (12-14). In cervical cancer, SIX1 is induced by the E7 oncoprotein of human papillomavirus, and is closely associated with tumorigenesis of cervical epithelium (15). Increased expression of SIX1 could promote tumor growth by accelerating the cell cycle process (15), and enhance the metastatic potential of cervical cancer cells by upregulating the expression of $\alpha 5 \beta 1$ integrin (16). Importantly, the present authors recently reported that SIX1 could amplify TGF $\beta$ signals, interacting with SMAD2/3 proteins to regulate gene expression (17). Based on these findings, the present study investigated whether SIX1 and TGF $\beta$ were responsible for modulating the EMT program in cervical cancer. The data obtained indicated 
that SIX1 could coordinate with TGF $\beta$ to induce EMT, thus enhancing the metastatic capacity of cervical cancer cells.

\section{Materials and methods}

Cells and transfection. The human cervical cancer cell line $\mathrm{SiHa}$ was purchased from the American Type Culture Collection (Manassas, VA, USA) and cultured in Dulbecco's modified Eagle's medium (DMEM; Hyclone; GE Healthcare Life Sciences, Beijing, China) supplemented with $10 \%$ fetal bovine serum (Hyclone; GE Healthcare Life Sciences) at $37^{\circ} \mathrm{C}$ and $5 \% \mathrm{CO}_{2}$. Cells were transduced with CMV-Fluc-IRES-RFP lentiviral particles (GeneChem, Co., Ltd., Shanghai China) expressing luciferase and red fluorescent protein (RFP). Cells with stable transfection, which expressed RFP, were isolated by fluorescence-activated cell sorting on a FACSCalibur flow cytometer and analyzed with CellQuest Pro 6.0 software (BD Biosciences, Franklin Lakes, NJ, USA).

SiHa cells were transfected with plasmid pcDNA3.1-control (Thermo Fisher Scientific, Inc., Waltham, MA, USA), which harboured neomycin-resistance, or with pcDNA3.1-SIX1, which was kindly donated by Dr Kong-Ming Wu (Department of Cancer Biology, Thomas Jefferson University, Philadelphia, PA, USA), using Lipofectamine 2000 (Invitrogen; Thermo Fisher Scientific, Inc.). The expression of TGF $\beta$ receptor 1 (T $\beta R 1)$ was knocked down using puromycin-resistance small hairpin (sh)RNA lentiviral particles (GeneChem, Co., Ltd.) targeting 5'-CTGTAATTCTGCTGTAATA-3'. As a negative control (NC), shNC, a shRNA not targeting any known gene, was used.Cells with stable transfection were selected with G418 and puromycin (Sigma-Aldrich, St. Louis, MO, USA). The transfected cells were designated as SiHa-control, SiHa-SIX1, SiHa-SIX1-shNC and SiHa-SIX1-shT $\beta R 1$. To downregulate the expression of SMAD2 and SMAD3, the corresponding small interfering (si)RNAs (Guangzhou RiboBio Co., Ltd., Guangzhou, China) were transfected into tumor cells using Lipofectamine 2000, according to the manufacturer's protocol.

Bioinformatic analysis. The normalized RNA-sequencing data of cervical cancer samples was publicly available from The Cancer Genome Atlas (TCGA) database (https://tcga-data.nci.nih.gov/tcga/tcgaHome2.jsp).Differential gene expression based on SIX1 expression level was determined as previously described (15) using Bioconductor edgeR software package (18), freely available at www.bioconductor.org. Differences were considered to be statistically significant when false discovery rate was $<0.25$.

Assay of gene expression by reverse transcription-quantitative polymerase chain reaction ( $R T-q P C R)$. Total RNA was extracted from cells with TRIzol reagent (Invitrogen; Thermo Fisher Scientific, Inc.). Total RNA was treated with Ambion Turbo DNA-free DNase (Thermo Fisher Scientific, Inc.; Austin, TX, USA), according to the manufacturer's instructions. RNA $(2 \mu \mathrm{g})$ was used for first-strand cDNA synthesis with reverse transcriptase (Promega Corporation, Madison, WI, USA), according to the manufacturer's protocol. cDNA was amplified by qPCR in a CFX96 Touch Real-Time PCR Detection system with CFX Manager 3.0 software (Bio-Rad Laboratories, Inc., Hercules, CA, USA) using iQ SYBR
Green Supermix (Bio-Rad Laboratories, Inc.). Glyceraldehyde 3-phosphate dehydrogenase (GAPDH) and eukaryotic translation elongation factor 1 alpha 1 (EEF1A1) were used as reference genes, since this was reported to be the most reliable gene combination in cervical cancer (19). The primer sequences for RT-qPCR were as follows: GAPDH, sense 5'-GACAGT CAGCCGCATCTTCT-3' and antisense 5'-TTAAAAGCAGC CCTGGTGAC-3'; EEF1A1, sense 5'-TGCGGTGGGTGTCAT CAAA-3' and antisense 5'-AGAGTGGGGTGGCAGGTA TTG-3'; SIX1, sense 5'-CACCAGTTCTCGCCTCACA-3' and antisense 5'-CACCCGATATTTGCCCAC-3'; E-cadherin, sense 5'-AGGCCAAGCAGCAGTACATT-3' and antisense 5'-ATTCACATCCAGCACATCCA-3'; and N-cadherin, sense 5'-CCATCAAGCCTGTGGGAATC-3' and antisense 5'-GCA GATCGGACCGGATACTG-3' (Beijing Qing Ke New Industrial Biotechnology Co., Ltd., Beijing, China). The reaction included $5 \mathrm{ng}$ cDNA and $10 \mathrm{pM}$ of each primer. The thermal cycling conditions for qPCR were as follows: Denaturation at $95^{\circ} \mathrm{C}$ for $10 \mathrm{~min} ; 40$ cycles of denaturation at $95^{\circ} \mathrm{C}$ for $10 \mathrm{sec}$, annealing at $60^{\circ} \mathrm{C}$ for $30 \mathrm{sec}$ and extension at $72^{\circ} \mathrm{C}$ for $30 \mathrm{sec}$. Gene expression was quantified using the comparative $\mathrm{Cq}$ method (19). The expression levels of each mRNA were normalized to those of GAPDH and EEF1A1 mRNAs, and expressed as $\mathrm{n}$-fold difference relative to the control.

Immunohistochemistry. Cervical squamous cell carcinoma samples were acquired from the Clinical Database and Biobank of Patients with Gynecologic Neoplasms (ClinicalTrials.gov Identifier: NCT01267851), with informed consent obtained from all subjects participating in the study. The samples were obtained by surgery from cancer patients without preoperative treatment between February 2007 and August 2009 at Tongji Hospital of Tongji Medical College (Wuhan, China). Tumor samples were processed into tissue microarrays (TMAs) at Shanghai Outdo Biotech Co., Ltd. (Shanghai, China). TMAs and tissue sections were subjected to immunohistochemical analysis using the Vectastain ABC kit (ZSGB-BIO, Beijing, China), according to the manufacturer's protocol. Rabbit polyclonal SIX1 antibody (1:200; catalogue no. HPA001893; Sigma-Aldrich) and rabbit monoclonal E-cadherin antibody (1:500; catalogue no. EP700Y; Abcam, Cambridge, UK) were used as primary antibodies. Low or high protein levels of SIX1 in tissue were evaluated as previously described (17). Since E-cadherin is expressed on the cellular membrane of normal cervical epithelium (3), positive membrane expression of E-cadherin was classified as normal, while significant reduction of membrane expression of E-cadherin was classified as a low-E-cadherin level.

Western blot assay. Western blot assay was performed as previously described (20). Briefly, the cells were harvested and lysed using ice-cold RIPA lysis buffer [50 mM Tris- $\mathrm{HCl}$ ( $\mathrm{pH} 7.4)$, $150 \mathrm{mM}$ sodium chloride, $1 \%$ Nonidet P-40 and $0.5 \%$ sodium deoxycholate; Google Wuhan Biological Technology Co., Ltd., Wuhan, China]. Following centrifugation at $10,000 \mathrm{x}$ g for $15 \mathrm{~min}$ at $4^{\circ} \mathrm{C}$, the proteins in the supernatants were quantified using Bradford method, separated on 10\% SDS-PAGE gel (Google Wuhan Biological Technology Co., Ltd.) and electrotransferred from the gel to a nitrocellulose membrane (Merck \& Co., Inc., Whitehouse Station, NJ, USA). After blocking with 
A

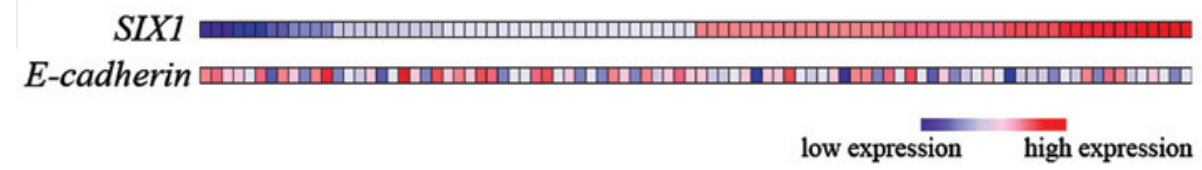

B

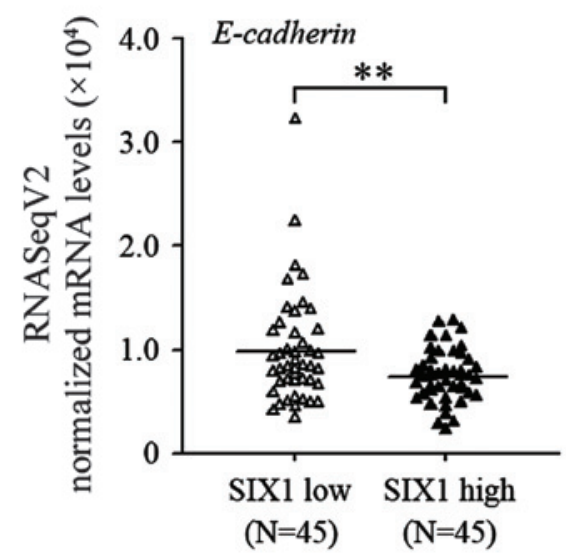

C

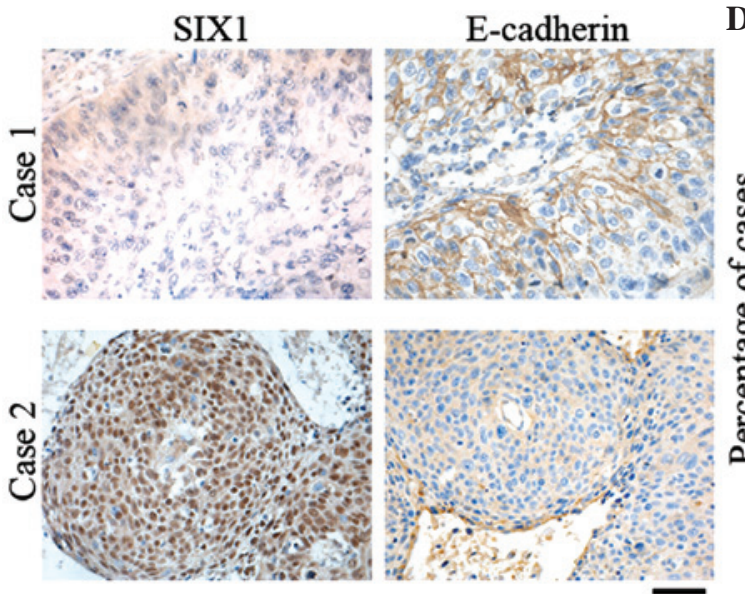

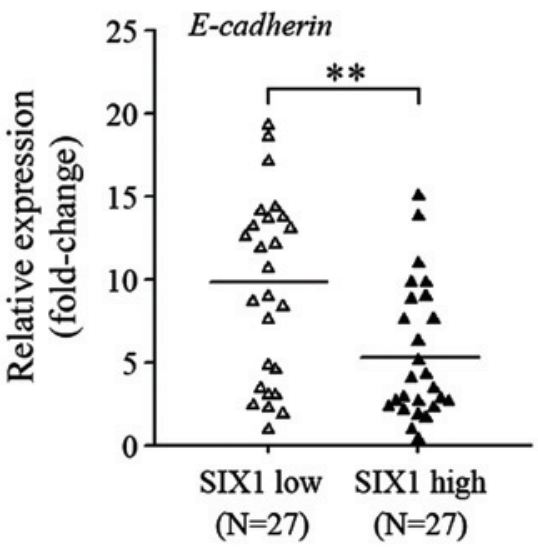

D
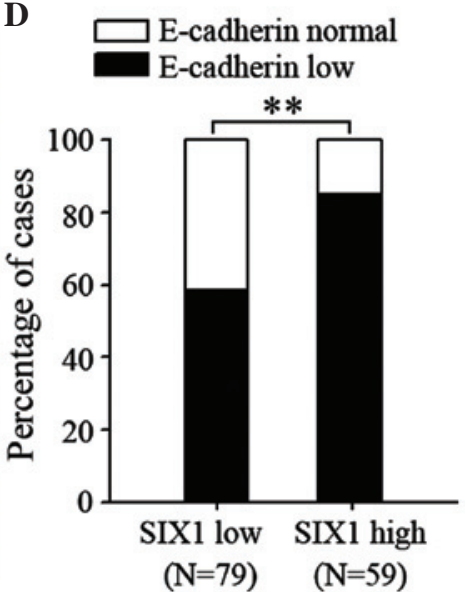

Figure 1. The expression of SIX1 and E-cadherin is negatively correlated in cervical cancer. (A) The expression profile of SIX1 and E-cadherin in cervical cancer specimens obtained from the TCGA database is represented as a heat map. (B) The TCGA RNA-sequencing data of cervical cancer was divided into two groups by the median level of SIX1 expression. The normalized messenger RNA levels of E-cadherin in the samples are indicated. ${ }^{* *}$ False discovery rate $<0.05$. The expression levels of SIX1 and E-cadherin in human CSCC specimens were detected by reverse transcription-quantitative polymerase chain reaction. (C and D) Immunohistochemical analysis of SIX1 and E-cadherin protein expression in tissue microarrays of human CSCC specimens. (C) Representative images of SIX1 and E-cadherin staining (magnification, x200; scale bar, $100 \mu \mathrm{m}$ ). (D) Percentage of cases with different intensity of E-cadherin staining. ${ }^{* *} \mathrm{P}<0.01$. TCGA, The Cancer Genome Atlas; SIX1, sine oculis homeobox homolog 1; mRNA, messenger RNA; RNASeqV2, RNA-sequencing version 2; CSCC, cervical squamous cell carcinoma.

$5 \%$ skimmed milk (Google Wuhan Biological Technology Co., Ltd.) in phosphate-buffered saline, the membranes were immunoblotted with the primary antibodies at $4^{\circ} \mathrm{C}$ overnight. The primary antibodies used were as follows: Anti-human SIX1 (1:1,100; catalogue no. HPA001893; Sigma-Aldrich), anti-E-cadherin (1:10,000; catalogue no. ab40772; Abcam), anti-N-Cadherin (1:1,000; catalogue no. ab18203; Abcam), anti-SMAD2 (1:1,000; catalogue no. 5339; Cell Signaling Technology, Inc., Danvers, MA, USA), anti-SMAD3 (1:1,000; catalogue no. 9523; Cell Signaling Technology, Inc.) and $\beta$-actin (1:500; catalogue no. ab8229; Abcam). Signals were detected using a SuperSignal West Pico Chemiluminescent Substrate kit (Pierce Biotechnology, Inc., Rockford, IL, USA) with a ChemiDoc XRS+ machine (Bio-Rad Laboratories, Inc.). Protein levels of $\beta$-actin were employed as loading controls. The relative protein expression levels were analyzed using Image Lab software (version 4.1; Bio-Rad Laboratories, Inc.).
Migration assay. Migration assay was performed using a Transwell chamber (Corning Incorporated, Corning, NY, USA). Tumor cells $\left(1 \times 10^{4}\right.$ cells) were placed in the upper chamber. Following 8-h incubation at $37^{\circ} \mathrm{C}$ in a humidified incubator with $5 \% \mathrm{CO}_{2}$, the cells that passed through the membrane were fixed with $4 \%$ paraformaldehyde and stained with $0.1 \%$ crystal violet (both from Google Wuhan Biological Technology Co., Ltd.) prior to be counted under a microscope (BX53; Olympus Corporation Tokyo, Japan). Cells from three randomly selected fields in each membrane were counted, and the average number of cells per field was calculated.

Animal studies. Female athymic nude (nu/nu) 4-week-old mice $(n=40)$ were purchased from Beijing HFK Bio-technology Co, Ltd. (Beijing, China). The studies were approved by the Committee on the Ethics of Animal Experiments of Tongji Medical College (Wuhan, China). Mice were 
A

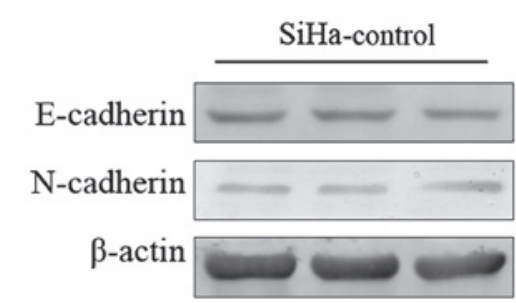

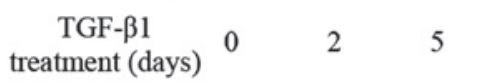

B

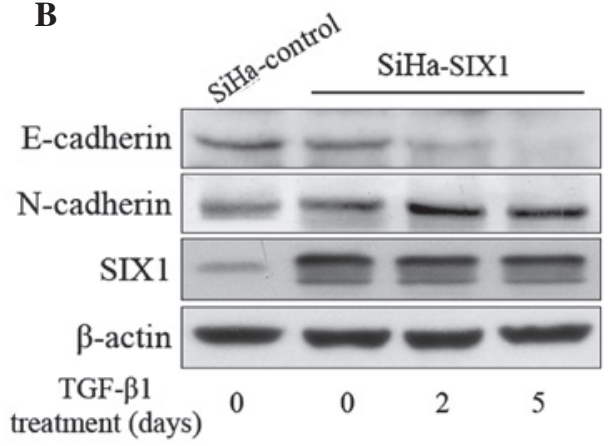

C

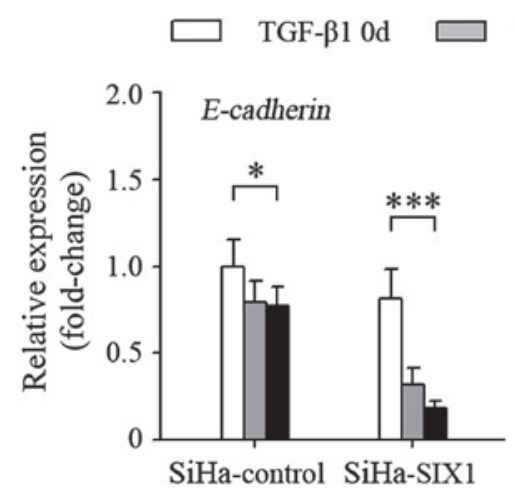

D

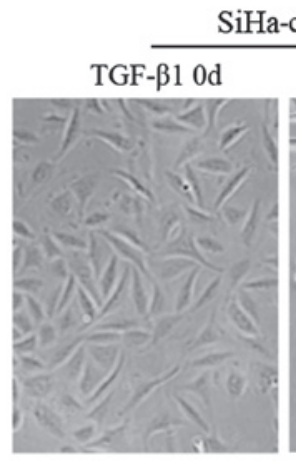

SiHa-control

TGF- $\beta 12 d$

TGF- $\beta 15 d$
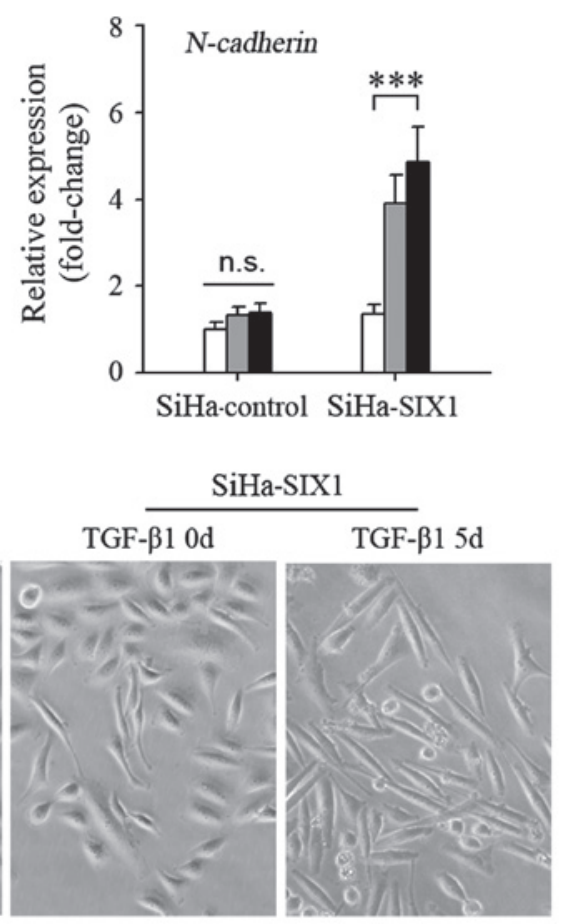

Figure 2. SIX1 enhances TGFß-induced epithelial-mesenchymal transition in cervical cancer cells. SiHa cells, untransfected or transfected with SIX1-expressing vector, were untreated or treated with TGF $\beta 1(1 \mathrm{ng} / \mathrm{ml})$ for the indicated times. The protein expression levels of E-cadherin and N-cadherin in (A) SiHa-control and (B) SiHa-SIX1 cells were detected by western blotting. The upper band is phosphorylated SIX and the lower band is unphosphorylated SIX1. Both bands were taken into account for phosphorylation. (C) The messenger RNA expression levels of E-cadherin and N-cadherin were detected by reverse transcription-quantitative polymerase chain reaction. (D) The morphological changes caused by SIX1 or/and TGF $\beta 1$ in SiHa cells were observed by microscopy. " $\mathrm{P}<0.05 ;{ }^{* * *} \mathrm{P}<0.001$. SIX1, sine oculis homeobox homolog 1 ; TGF; transforming growth factor; n.s., not significant.

maintained in the accredited animal facility of Tongji Medical College at $20-26^{\circ} \mathrm{C}$ in a 12 -h light/dark cycle. A lymphatic metastasis model of cervical cancer was used as previously described (16). Briefly, $5 \times 10^{6}$ tumor cells in $50 \mu 1$ serum-free DMEM (Boster Biological Technology, Co., Ltd., Wuhan, China)/Matrigel (BD Biosciences) at a 9:1 ratio were injected subcutaneously into the claw pads of the mice. Tumor size $\left(\mathrm{mm}^{3}\right)$ was measured and calculated by the following formula: Volume $=(\text { width })^{2} \mathrm{x}$ length $/ 2$. When primary tumors reached $\sim 150 \mathrm{~mm}^{3}$ in size, metastases were tracked by optical imaging of luciferase activity originating from tumor cells using the IVIS Spectrum system (Caliper Life Sciences; PerkinElmer, Inc., Waltham, MA, USA). Subsequently, when primary tumors reached $\sim 250 \mathrm{~mm}^{3}$ in size, the mice were euthanized, and their popliteal and inguinal lymph nodes were excised. Metastases of tumor cells in the lymph nodes were confirmed by detection of tumor-expressed RFP under a SZX16 dissecting microscope (Olympus Corporation). The incidence of metastasis-positive mice in each group was calculated.

Statistical analysis. Each experiment was independently repeated $\geq 3$ times. SPSS version 13.0 software (SPSS, Inc., Chicago, IL, USA) was used for statistical analysis. The results were expressed as the mean \pm standard deviation, and interpreted by one-way analysis of variance or $\chi^{2}$ test. $\mathrm{P}<0.05$ was considered to indicate a statistically significant difference.

\section{Results}

SIX1 expression is associated with EMT of cervical cancer. To determine the effect of SIX1 on EMT of cervical cancer, the effect of SIX1 on the expression of E-cadherin, which is a 
A

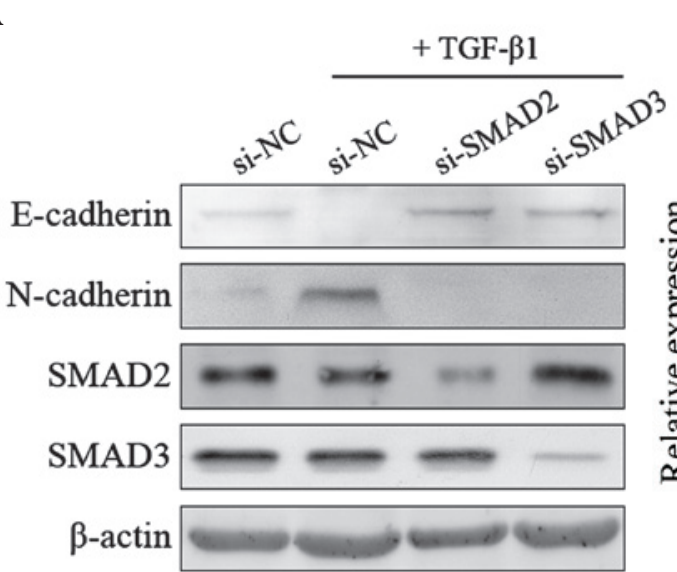

B

$\square$ si-NC

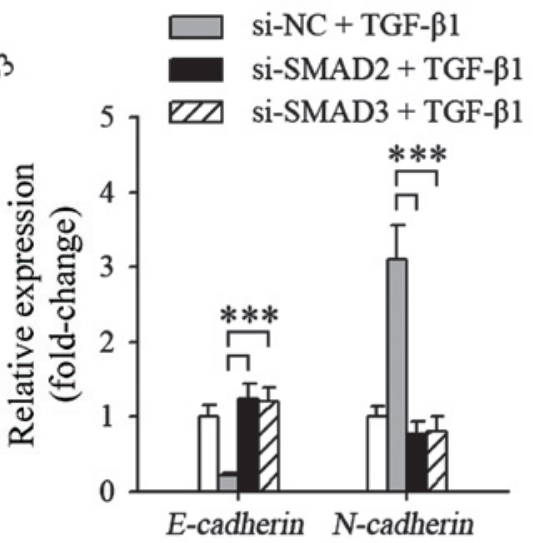

C
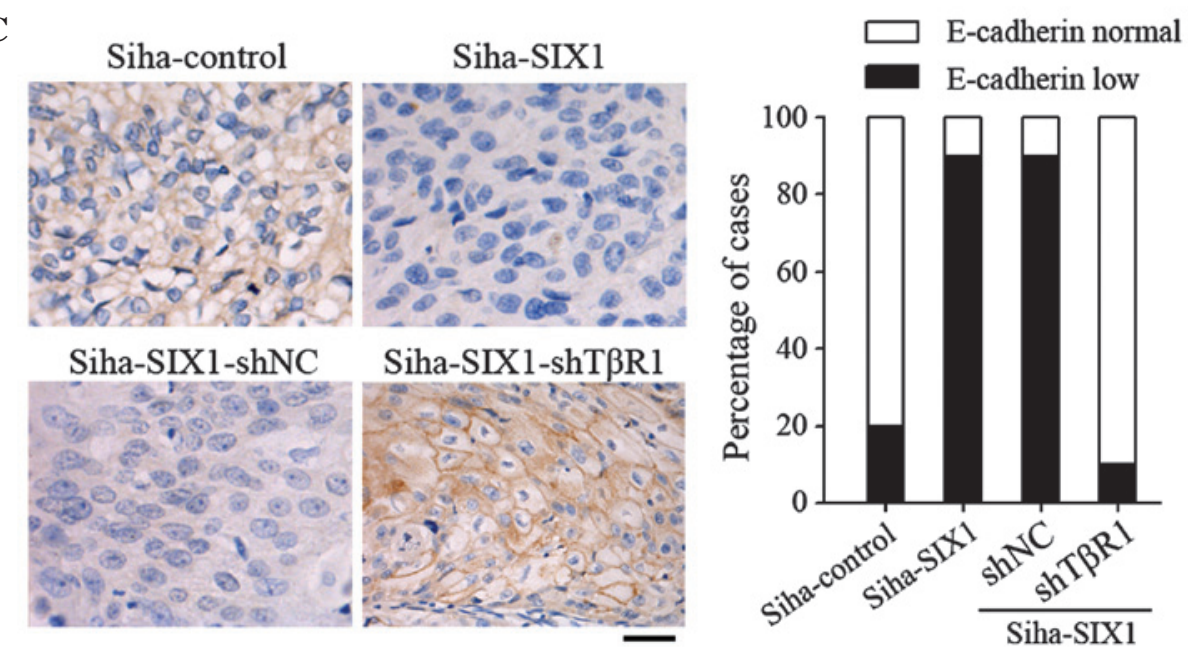

Figure 3. TGF $\beta$ signals are necessary for SIX1 to induce epithelial-mesenchymal transition in cervical cancer. (A and B) At $24 \mathrm{~h}$ post-transfection with the indicated small interfering RNAs, the cells were stimulated with TGF $\beta 1(1 \mathrm{ng} / \mathrm{ml})$ for 5 days. The expression levels of E-cadherin and N-cadherin were detected by (A) western blotting or (B) reverse transcription-quantitative polymerase chain reaction. (C) SiHa cells were injected subcutaneously into the claw pads of mice to form primary tumors, and the protein expression levels of E-cadherin in the tumors were analyzed by immunohistochemical analysis. Left panel, representative images of SIX1 and E-cadherin staining of primary tumors (magnification, x400; scale bar, $50 \mu \mathrm{m}$ ). Right panel, percentage of cases ( $\mathrm{n}=10$ /group) with different intensity of E-cadherin staining. ${ }^{* * * *} \mathrm{P}<0.001$. TGF; transforming growth factor; SIX1, sine oculis homeobox homolog 1 ; NC, negative control; sh, small hairpin; si, small interfering; T $\beta R 1$, TGF $\beta$ receptor 1.

common feature of EMT, was analyzed. The RNA-sequencing data of cervical cancer specimens was obtained from the TCGA database. The expression profiles of SIX1 and E-cadherin are shown in Fig. 1A. The specimens were divided into SIX1-low expression group and SIX1-high expression group. Using Bioconductor edgeR software package, E-cadherin was identified to be one of the significantly downregulated genes in the SIX1-high expression group (false discovery rate $=0.046$; Fig. 1B). These results were further confirmed by quantifying the mRNA levels of SIX1 and E-cadherin in the samples $(\mathrm{P}=0.003$; Fig. 1C). A significant decrease in the membrane expression levels of E-cadherin was observed at the primary site of cervical cancer samples that highly expressed SIX1 $(\mathrm{P}=0.001$; Fig. 1D). These results suggest that increased SIX1 expression is associated with mesenchymal phenotype of cervical cancer.

SIX1 enhances TGF $\beta$-induced EMT in cervical cancer cells. TGF $\beta$ is one of the most important inducers of EMT $(5,21)$. However, treatment with TGF $\beta 1$ only suppressed slightly the expression of the epithelial marker E-cadherin, and negligibly induced the expression of the mesenchymal marker $\mathrm{N}$-cadherin in SiHa cells, a cervical cancer cell line with low expression levels of SIX1 (Fig. 2A) (17). These results suggested that cervical cancer cells may not be sensitive to TGF $\beta 1$. Overexpression of SIX1 in SiHa cells affected slightly the expression of E-cadherin and N-cadherin (Fig. 2B). Notably, TGF $\beta 1$ could effectively induce SIX1-expressing tumor cells to undergo EMT (Fig. 2B). Similar results were obtained when E-cadherin (SiHa-control 0 vs. 5 days, $\mathrm{P}=0.016$; SiHa-SIX1 0 vs. 5 days, $\mathrm{P}<0.001)$ and $\mathrm{N}$-cadherin (SiHa-control 0 vs. 5 days, $\mathrm{P}=0.071$; SiHa-SIX1 0 vs. 5 days, $\mathrm{P}<0.001$ ) expression was quantified using RT-qPCR (Fig. 2C). Correspondingly, TGF $\beta 1$-treated SiHa-SIX1 cells became more fusiform with decreased connection between the cells than TGF 31 -treated SiHa-control cells (Fig. 2D). These results demonstrate that SIX1 coordinates with TGF $\beta$ to induce EMT in cervical cancer cells.

TGF $\beta$ signals are required for SIXI-induced EMT. The present authors previously reported that SIX1 could interact with SMAD proteins, which are the signal transducers of 
A
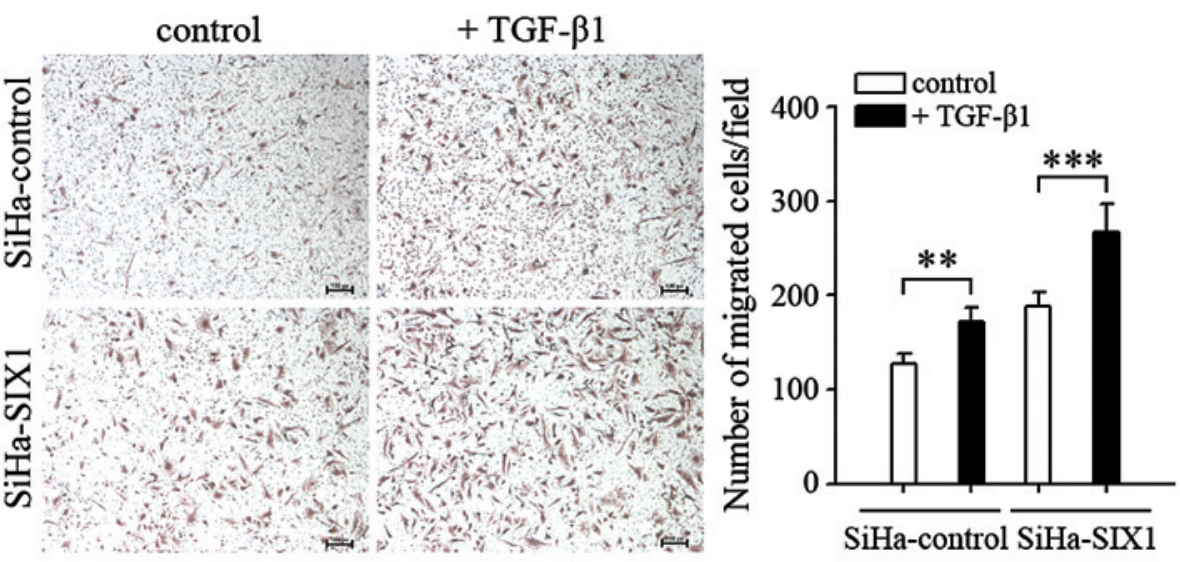

B

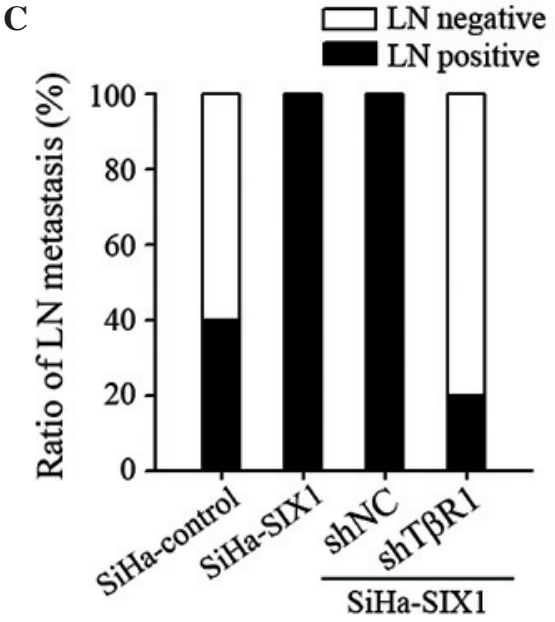

Figure 4. SIX1 coordinates with TGF $\beta$ signals to enhance the metastatic capacity of cervical cancer cells. (A) Migration assay. SiHa cells, untransfected or transfected with SIX1-expressing vector, were untreated or treated with TGF $\beta 1(1 \mathrm{ng} / \mathrm{ml})$ for 5 days, and their migration ability was assessed (scale bar, $100 \mu \mathrm{m}$ ). (B) In vivo bioluminescence and fluorescence representative images of lymphatic metastasis in mice. Lymph nodes in the popliteal and inguinal regions of the mice were detected by bioluminescence and fluorescence signals, corresponding to luciferase activity and red fluorescent protein expression, respectively. The black arrow indicates tumor cells inside the lymph node, while the arrowhead indicates a tumor-invaded lymphatic vessel. (C) The ratios of lymph node metastasis were calculated ( $\mathrm{n}=10$ /group). ${ }^{* *} \mathrm{P}<0.01 ;{ }^{* * *} \mathrm{P}<0.001$. SIX1, sine oculis homeobox homolog 1; TGF; transforming growth factor; LN, lymph node; T $\beta \mathrm{R} 1$, TGF $\beta$ receptor $1 ; \mathrm{NC}$, negative control; sh, small hairpin.

TGF $\beta$ signaling (17). To further clarify the role of TGF $\beta$ signals in inducing EMT on SIX1-expressing tumor cells, the components of the TGF $\beta$ signaling pathway were inhibited in the present study. When the expression of SMAD2 or SMAD3 was knocked down, the TGF $\beta 1$-induced EMT process in SiHa-SIX1 cells was abrogated (Fig. 3A). The same results were obtained when E-cadherin (si-SMAD2, P<0.001; si-SMAD3, $\mathrm{P}<0.001)$ and $\mathrm{N}$-cadherin (si-SMAD2, $\mathrm{P}<0.001$; si-SMAD3, $\mathrm{P}<0.001$ ) expression was quantified using RT-qPCR (Fig. 3B). Albeit not significantly induced EMT in vitro, overexpression of SIX1 alone resulted in a decrease in E-cadherin expression in vivo $(\mathrm{P}=0.002$; Fig. $3 \mathrm{C})$. However, silencing T $\beta \mathrm{R} 1$ completely abrogated the effect of SIX1 ( $<<0.001$; Fig. 3C), further indicating that TGF $\beta$ signals are required for SIX1 to induce EMT.

SIXI coordinates withTGF $\beta$ to enhance the metastatic capacity of cervical cancer cells. EMT is associated with the metastatic capacity of tumor cells $(5,6)$. Based on the above results, the effect of SIX1 and TGF $\beta 1$ on the metastatic phenotype of cervical cancer cells was further investigated. The migration of SiHa cells was increased following TGF $\beta 1$ stimulation.
TGF $\beta 1$ was more efficient than the control in promoting the migratory capacity of tumor cells if the cells expressed high levels of SIX1 (SiHa-control, P=0.003; SiHa-SIX1, P<0.001; Fig. 4A). The metastatic capacity of tumor cells was further tested using a previously described lymphatic metastasis model (Fig. 4B). The results indicated that SIX1-mediated promotion of lymph node metastasis was completely abolished by silencing T $\beta R 1$ (SiHa-SIX1 shNC vs. SiHa-SIX1 shT $\beta R 1$, $\mathrm{P}=2.61 \mathrm{E}-4$; Fig. 4C). These results demonstrate that SIX1 and TGF $\beta$ signals coordinate to enhance the metastatic capacity of cervical cancer cells.

\section{Discussion}

An increasing number of studies have provided strong evidence for the critical role of the EMT program in tumor progression and metastasis $(5,6)$. Although TGF $\beta$ is regarded as one of the most important inducers of EMT, several non-invasive tumor cells were not able to undergo TGF $\beta$-induced EMT in vitro (9). The present data suggest that the expression levels of the transcription factor SIX1 could determine the sensitivity of tumor cells to TGF $\beta$ stimulation. 
In the present study, the expression of SIX1 was negatively correlated with that of the epithelial marker E-cadherin in cervical cancer. Consistently, increased SIX1 expression in tumor cells could sufficiently reduce E-cadherin expression and promote lymph node metastasis in vivo. However, increased SIX1 expression in SiHa cells could not significantly induce EMT in vitro, suggesting that SIX1 could not efficiently induce EMT by itself. The present data demonstrated that the main contribution of SIX1 to EMT was to increase the sensitivity of tumor cells to TGF $\beta$ stimulation. Increased expression of SIX1 could promote EMT of cervical cancer cells in a TGF $\beta$-dependent manner. Based on the overexpression of SIX1, TGF $\beta$ induced more remarkable changes in the transition of phenotype than the SiHa-control group. Therefore, SIX1 and TGF $\beta$ coordinated to promote cell motility and tumor metastasis of cervical cancer.

Previous studies have described the roles of TGF $\beta$-activated SMADs in EMT. Increased expression of SMAD2 or SMAD3 was reported to induce EMT, whereas expression of dominant negative versions of SMAD2 or SMAD3 blocked TGF $\beta$-induced EMT (7,22). Additionally, TGF $\beta$ activates SMAD and non-SMAD signals, including Rho-like guanosine triphosphatases, phosphatidylinositol-4,5-bisphosphate 3 -kinase and mitogen-activated protein kinase signaling pathways, which also contribute to $\operatorname{EMT}(6,23,24)$. In the present study, TGF $\beta$ could efficiently induce EMT in vitro and lymph node metastasis in vivo if tumor cells expressed high levels of SIX1. However, knocking down the expression of SMAD2 or SMAD3 could completely block the EMT process induced by SIX1 and TGF $\beta$. In a previous study, the present authors demonstrated that SIX1 was involved in the SMAD2/3 protein complex and could enhance TGF $\beta$-SMAD signaling (17). Therefore, enhancing TGF $\beta$-SMAD signaling is an important mechanism by which SIX1 promotes EMT and lymphatic metastasis in cervical carcinoma.

The signal transduction of TGF $\beta$ forms a complex network, involving the activation of SMAD and non-SMAD signals, and the crosstalk among various signal transduction pathways such as wingless-related integration site and Notch signaling pathways provides context-dependent effects during EMT $(25,26)$. Therefore, context-specific outcomes may be generated according to the availability of repressors/activators, distinct intensity or duration of SMAD/non-SMAD signaling activity or changes in the levels of interacting protein partners (7). The present data revealed that TGF $\beta 1$ stimulation was not able to induce EMT in SiHa cells, which are cervical cancer cells with low expression of SIX1 (17). By contrast, when SiHa-SIX1 cells were stimulated with TGF $\beta 1$, significant changes were observed in the expression of EMT markers, cell morphology and metastatic capacity of the cells. In addition, higher expression levels of E-cadherin in SIX1-low clinical samples and in vivo experiments also suggested that environmental factors such as TGF $\beta$ were ineffective in inducing EMT in a SIX1-low context. Therefore, the coordination of SIX1 and TGF $\beta$ may be critical for inducing EMT in cervical carcinoma.

In summary, the present study revealed the important role of the coordination of SIX1 and TGF $\beta$ in inducing EMT in cervical cancer. SIX1 induced EMT of cervical cancer cells in a TGF $\beta$-dependent manner, and increased SIX1 expression significantly enhanced the TGF $\beta$-induced transition of mesenchymal phenotype, indicating that SIX1 and TGF $\beta$ coordinate to promote the EMT program and the metastatic capacity of cervical cancer cells. In conclusion, the present results demonstrate that SIX1 plays a crucial role in the progression and metastasis of cervical cancer, and suggest that targeting SIX1 and/or TGF $\beta$ signaling may be a valuable strategy in cancer therapy.

\section{Acknowledgements}

The present authors would like to thank Dr Qi-Lin Ao and Dr Shuang Guo (Department of Pathology, Union Hospital, Tongji Medical College, Huazhong University of Science and Technology, Wuhan, China) for reviewing the histology data. The present study was supported by the National Science Foundation of China (Beijing, China; grant nos. 81072135 and 81372801).

\section{References}

1. Gao D, Vahdat LT, Wong S, Chang JC and Mittal V: Microenvironmental regulation of epithelial-mesenchymal transitions in cancer. Cancer Res 72: 4883-4889, 2012.

2. Tsai JH and Yang J: Epithelial-mesenchymal plasticity in carcinoma metastasis. Genes Dev 27: 2192-2206, 2013.

3. Li Y, Wang W, Wang W, Yang R, Wang T, Su T, Weng D, Tao T, Li W, Ma D and Wang S: Correlation of TWIST2 up-regulation and epithelial-mesenchymal transition during tumorigenesis and progression of cervical carcinoma. Gynecol Oncol 124: 112-118, 2012.

4. Zhou XM, Zhang H and Han X: Role of epithelial to mesenchymal transition proteins in gynecological cancers: Pathological and therapeutic perspectives. Tumour Biol 35: 9523-9530, 2014.

5. Thiery JP, Acloque $\mathrm{H}$, Huang RY and Nieto MA: Epithelial-mesenchymal transitions in development and disease. Cell 139: 871-890, 2009.

6. Lamouille S, Xu J and Derynck R: Molecular mechanisms of epithelial-mesenchymal transition. Nat Rev Mol Cell Biol 15: 178-196, 2014.

7. Xu J, Lamouille S and Derynck R: TGF-beta-induced epithelial to mesenchymal transition. Cell Res 19: 156-172, 2009.

8. Katsuno Y, Lamouille S and Derynck R: TGF- $\beta$ signaling and epithelial-mesenchymal transition in cancer progression. Curr Opin Oncol 25: 76-84, 2013.

9. Brown KA, Aakre ME, Gorska AE, Price JO, Eltom SE, Pietenpol JA and Moses HL: Induction by transforming growth factor-beta1 of epithelial to mesenchymal transition is a rare event in vitro. Breast Cancer Res 6: R215-R231, 2004.

10. Feng XX, Liu M, Yan W, Zhou ZZ, Xia YJ, Tu W, Li PY and Tian DA: $\beta 3$ integrin promotes TGF- $\beta 1 / \mathrm{H}_{2} \mathrm{O}_{2} / \mathrm{HOCl}$-mediated induction of metastatic phenotype of hepatocellular carcinoma cells by enhancing TGF- $\beta 1$ signaling. PLoS One 8: e79857, 2013.

11. Ford HL, Landesman-Bollag E, Dacwag CS, Stukenberg PT, Pardee AB and Seldin DC: Cell cycle-regulated phosphorylation of the human SIX1 homeodomain protein. J Biol Chem 275: 22245-22254, 2000

12. Micalizzi DS, Christensen KL, Jedlicka P, Coletta RD, Barón AE, Harrell JC, Horwitz KB, Billheimer D, Heichman KA, Welm AL, et al: The Six1 homeoprotein induces human mammary carcinoma cells to undergo epithelial-mesenchymal transition and metastasis in mice through increasing TGF-beta signaling. J Clin Invest 119: 2678-2690, 2009.

13. Ng KT, Man K, Sun CK, Lee TK, Poon RT, Lo CM and Fan ST: Clinicopathological significance of homeoprotein Six1 in hepatocellular carcinoma. Br J Cancer 95: 1050-1055, 2006.

14. Behbakht K, Qamar L, Aldridge CS, Coletta RD, Davidson SA, Thorburn A and Ford HL: Six1 overexpression in ovarian carcinoma causes resistance to TRAIL-mediated apoptosis and is associated with poor survival. Cancer Res 67: 3036-3042, 2007.

15. Liu D, Zhang XX, Xi BX, Wan DY, Li L, Zhou J, Wang W, Ma D, Wang $\mathrm{H}$ and Gao QL: Sine oculis homeobox homolog 1 promotes DNA replication and cell proliferation in cervical cancer. Int J Oncol 45: 1232-1240, 2014. 
16. Liu D, Zhang XX, Wan DY, Xi BX, Ma D, Wang H and Gao QL: Sine oculis homeobox homolo 1 promotes $\alpha 5 \beta 1$-mediated invasive migration and metastasis of cervical cancer cells. Biochem Biophys Res Commun 446: 549-554, 2014.

17. Liu D, Li L, Zhang XX, Wan DY, Xi BX, Hu Z, Ding WC, Zhu D, Wang XL, Wang W, et al: SIX1 promotes tumor lymphangiogenesis by coordinating TGF $\beta$ signals that increase expression of VEGF-C. Cancer Res 74: 5597-5607, 2014.

18. Robinson MD, McCarthy DJ and Smyth GK: edgeR: A Bioconductor package for differential expression analysis of digital gene expression data. Bioinformatics 26: 139-140, 2010.

19. Shen Y, Li Y, Ye F, Wang F, Lu W and Xie X: Identification of suitable reference genes for measurement of gene expression in human cervical tissues. Anal Biochem 405: 224-229, 2010.

20. Wang C, Zhou L, Li S, Wei J, Wang W, Zhou T, Liao S, Weng D, Deng D, Weng Y, et al: C4orf7 contributes to ovarian cancer metastasis by promoting cancer cell migration and invasion. Oncol Rep 24: 933-939, 2010.

21. Thiery JP: Epithelial-mesenchymal transitions in tumour progression. Nat Rev Cancer 2: 442-454, 2002.
22. Valcourt U, Kowanetz M, Niimi H, Heldin CH and Moustakas A: TGF-beta and the Smad signaling pathway support transcriptomic reprogramming during epithelial-mesenchymal cell transition. Mol Biol Cell 16: 1987-2002, 2005.

23. Derynck R and Zhang YE: Smad-dependent and Smad-independent pathways in TGF-beta family signalling. Nature 425: 577-584, 2003.

24. Lamouille S and Derynck R: Emergence of the phosphoinositide 3-kinase-Akt-mammalian target of rapamycin axis in transforming growth factor- $\beta$-induced epithelial-mesenchymal transition. Cells Tissues Organs 193: 8-22, 2011.

25. Thiery JP and Sleeman JP: Complex networks orchestrate epithelial-mesenchymal transitions. Nat Rev Mol Cell Biol 7: 131-142, 2006

26. Timmerman LA, Grego-Bessa J, Raya A, Bertrán E, Pérez-Pomares JM, Díez J, Aranda S, Palomo S, McCormick F, Izpisúa-Belmonte JC and de la Pompa JL: Notch promotes epithelial-mesenchymal transition during cardiac development and oncogenic transformation. Genes Dev 18: 99-115, 2004. 